\title{
Anti-tick microbiota vaccines: how can this actually work?
}

\author{
Alejandra Wu-Chuang ${ }^{1} \cdot$ Dasiel Obregon $^{2} \cdot$ Lourdes Mateos-Hernández $^{1} \cdot$ Alejandro Cabezas-Cruz $^{1}$ (I)
}

Received: 16 April 2021 / Accepted: 8 June 2021 / Published online: 28 June 2021

(C) Institute of Zoology, Slovak Academy of Sciences 2021

\begin{abstract}
The main importance of ticks resides in their ability to harbor and transmit microorganisms that cause disease to animals and humans. In addition to pathogens, ticks coexist and interact with symbionts, and commensal bacteria that together form an ecological unit, the tick holobiont. The holobiont is an additional organismal level on which natural selection operates. The components of the tick hologenome, composed of host and microbiome genomes, are complimentary. In agreement with this proposition, antibiotic treatment produces disturbance of the tick-microbiota homeostasis which in turn decreases tick fitness and affects tick-pathogen interactions. Accordingly, we hypothesized that immune targeting of key members of the bacterial community of the tick microbiome by host antibodies could cause microbial dysbiosis with consequences for tick physiology and vector competence. Anti-tick microbiota vaccines were recently introduced as a tool to target the microbiota of vector arthropods by immunizing the vertebrate hosts against live keystone bacteria or tick microbiota bacterial proteins. This tool can also be used to target tick endosymbionts. Decreased abundance of selected keystone bacteria and/or endosymbionts may reshape the structure of tick microbial communities in a predictable manner. This tool can be used to manipulate the tick microbiome against ticks and transmitted pathogens. In this opinion, we explore the possibilities of this methodology for the control of ticks and tick-borne diseases.
\end{abstract}

Keywords Anti-tick microbiota vaccine $\cdot$ Tick microbiome $\cdot$ Keystone bacteria

\section{The tick microbiome: a gate to tick physiology and vector competence}

Tick microbiome has acquired importance in recent years becoming the focus of study by several research groups aiming to establish its functional role. Mounting evidence suggests that tick microbiome is not an inactive unit, but an interactive component of tick biology that can impact tick physiology and interestingly, vector competence (Narasimhan and Fikrig 2015; Wu-Chuang et al. 2021). Several studies have shown that a reduced bacterial load was associated with decreased reproductive fitness after antibiotics treatment in ticks (Zhong et al. 2007; Clayton et al. 2015; Zhang et al. 2017; Ben-Yosef et al. 2020). Specifically, a correlation between decreased fitness and reduced number of Coxiella-like endosymbionts (CLE) in Amblyomma americanum (Linnaeus, 1758; Zhong

Alejandro Cabezas-Cruz

alejandro.cabezas@vet-alfort.fr

1 Anses, INRAE, Ecole Nationale Vétérinaire d'Alfort, UMR BIPAR, Laboratoire de Santé Animale, Maisons-Alfort F-94700, France

2 School of Environmental Sciences, University of Guelph, Ontario Guelph N1G 2W1, Canada et al. 2007), Haemaphysalis longicornis (Neumann, 1901; Zhang et al. 2017) and Rhipicephalus sanguineus (Latreille, 1806; Ben-Yosef et al. 2020) have been reported, suggesting that CLE is an important bacterium in tick physiology. Several studies in Ixodes scapularis (Say, 1821) have found an association between Borrelia burgdorferi, the causative agent of Lyme disease, and the abundance of rickettsial endosymbionts (Kwan et al. 2017), Sphingomonas (Landesman et al. 2019), Tepidomonas, Luteibacter, Francisella, Fibriimonas (Brinkerhoff et al. 2020), Bacillus, Enterobacteriaceae and Pseudomonas (Ross et al. 2018). Although the mechanisms involved in the modulation of vector competence by tick microbiome are not well understood, Narasimhan et al. (2014) showed the existence of a functional link between the tick gut microbiota, the signal transducer and activator of transcription (STAT) signaling pathway and pathogen colonization. Specifically, these authors showed that I. scapularis larvae reared under 'sterile' conditions presented perturbed gut microbiota and reduced spirochetes colonization compared to larvae reared in normal conditions. Furthermore, larvae with perturbed microbiota presented decreased expression of STAT and peritrophin, a glycoprotein of the peritrophic matrix (PM), resulting in an altered tick gut peritrophic membrane integrity and consequently, in decreased epithelium- 
bound spirochete. Similarly, Yang et al. (2021) showed that the inhibition of a novel PM associated protein, Peritrophic Membrane Chitin Binding Protein (PM-CBP), altered PM integrity in I. scapularis and hence, altered gut microbiota, $B$. burgdorferi colonization and its transmission to mice. These results suggest that $B$. burgdorferi infection in ticks needs a particular gut microbial environment to maintain the PM integrity and therefore facilitate pathogen colonization. The crosstalk between tick microbiota and tick gut structure and its influence in tick-borne pathogens was also observed for Anaplasma phagocytophilum, the agent of human granulocytic anaplasmosis. In this case, Abraham et al. (2017) showed that A. phagocytophilum induces the expression of I. scapularis antifreeze glycoprotein (IAGFP) which perturbs tick microbiota, decreases PM thickness, alters gut permeability and the capacity of bacteria biofilms formation. In contrast to B. burgdorferi, A. phagocytophilum needs a disturbed PM for successful colonization. These results suggest the existence of a link between tick microbiome and tick gut whereby specific tick microbiota composition can modulate the integrity and the functional role of tick gut barrier and hence influences tick-borne pathogens colonization. The use of broad spectrum antimicrobial compounds, such as antibiotics, targeting several bacterial species does not allow establishing causal links between the reduction of specific taxa abundance in tick microbiota and changes in tick physiology or pathogen colonization. The lack of tools for the precise manipulation of the tick microbiome is currently a major limitation to achieve mechanistic insights into the tick microbiome (Narasimhan et al. 2021).

\section{How to identify network hubs and what is their relevance for keystone taxa inference?}

Disentangling microbe-microbe interactions is an essential step toward understanding the functional roles of the microbiome and its impact on host physiology and pathogen infection. The study of host-pathogen-microbiome interactions may lead to the development of therapeutic interventions to prevent microbial infections (Fisher and Mehta 2014; Layeghifard et al. 2017). Network analytical methods can be used to explore microbial interaction patterns in highly complex microbial communities such as those found in the microbiome (Faust and Raes 2012). A wide range of methods (e.g., distance- and correlation-based techniques) have been used to infer microbial networks, with varying levels of efficiency and accuracy (Beiko et al. 2018). Co-occurrence networks can reveal positive or negative interactions, underlaying cooperation (e.g., transfer of complementary metabolites or quorum sensing) or antagonism (competition for a limiting resource or direct interference) between microorganisms, respectively (Berry and Widder 2014). Co-occurrence networks have been successfully used to exploring the tick microbiome in response to disturbance (Estrada-Peña et al. 2020a, b). Within these networks, nodes represent taxa and edges represent correlations, hence the number of edges (grade) indicate the connectedness of each node in the network. Highly connected nodes are referred to as "hub" nodes, which are essential for maintaining network structure (i.e., if removed, the network can collapse) (Faust and Raes 2012). Hub nodes underscore key functional roles within the networks (Berry and Widder 2014; van der Heijden and Hartmann 2016). In human and plant microbiota, hub taxa may exert high influence on the diversity of the metacommunity by acting indirectly (via the host) or directly (via metabolites) on the colonization of other microbes (Fisher and Mehta 2014; Agler et al. 2016).

Network centrality metrics (e.g., degree centrality, betweenness, closeness and eigenvector-centrality) (Newman 2010) are often used to identify these hub taxa, however, there is currently no consensus in the best methods for their identification. For instance, some studies highlighted as key taxa those with maximum betweenness centrality scores (i.e., reflects the function of a node as bridge connecting modules in a network) (Banerjee et al. 2016; Ho et al. 2020), whereas other studies suggested that keystone species tends to have a combination of low betweenness centrality values, and high mean degree, or high closeness centrality, and high transitivity (Berry and Widder 2014; Banerjee et al. 2019a, b). Three main categories encompassed the methods used to identify network hubs: $i$ ) centrality indices, $i i$ ) node influence metrics, and iii) link analysis (i.e., page rank and hub score algorithms) methods (Layeghifard et al. 2017), which should be used based on the aims of each study. Among them, network hubs are promising candidates for the inference of keystones in microbial communities, but not all network hubs are keystone taxa in real life microbial communities (Agler et al. 2016). Of note, other authors have questioned the suitability of network hubs to infer keystone taxa altogether (Banerjee et al. 2019a, b; Röttjers and Faust 2019). Predicted network hubs requires experimental validation to uncover their biological importance as keystones (Banerjee et al. 2019a, b; Zheng et al. 2021). Experimental validation of keystones often involves comparing the effects of removal and/or addition of keystone candidate and other community members ( $\mathrm{Ze}$ et al. 2012; Röttjers and Faust 2019).

Some of this theoretical considerations were empirically tested by Mateos-Hernández et al. (2020), who identified hub taxa in tick microbiome using eigenvector-centrality (i.e., indicates the connectivity of the node with other well connected nodes in the network (Ruhnau 2000)) values as main criteria to predict keystoneness. In their recent studies, Mateos-Hernández et al. $(2020,2021)$ combined the eigenvector-centrality metric with analisis of relative abundance and ubiquitiousnes for the identification of network hubs and prediction of keystone taxa in the tick microbiome. 
The functional role of one of the network hubs identified (i.e., family Enterobacteriaceae) was validated using antimicrobiota vaccines in vivo. Immune targeting of the network hub within the ticks impacted tick fitness (MateosHernández et al. 2020, 2021) and microbial community structure (Mateos-Hernández et al. 2021).

\section{Antimicrobiota vaccines, a precision microbiology tool for tick microbiome manipulation}

Anti-tick microbiota vaccines were recently developed as a precision microbiology tool to modulate the tick microbiome in a taxon-specific manner (Mateos-Hernández et al. 2020, 2021). Combining 16 S rRNA amplicon sequencing and network analysis, keystone taxa were identified in the tick microbiome and used to target microbiota bacteria in ticks fed on mice immunized with a live bacteria vaccine containing the selected taxa (Fig. 1). The abundance of the keystone bacteria decreased in ticks fed on immunized mice. Microbiome modulation by antimicrobiota vaccines was also associated with decreased tick microbiome diversity (Mateos-Hernández et al. 2021). Modulation of vector microbiome was mediated by host antibodies (Mateos-Hernández et al. 2021), which together with complement proteins not only retain their immune functions inside the ticks, but also access broadly tick tissues including the midguts and salivary glands (Ackerman et al. 1981; Ben-Yakir et al. 1987; Wang and Nuttall 1994; Willadsen 1997; Rathinavelu et al. 2003; Galay et al. 2018). Notably, no mortality was associated with antimicrobiota immunization and no sign of pain was observed after the vaccination in the mice (Mateos-Hernández et al. 2020, 2021). Host immunization against keystone bacteria can be used to trigger the production of bacteria-specific antibodies that can target specific tick microbiota bacteria and affect tick fitness and/or pathogen transmission. Antimicrobiota vaccines offer the possibility to target specific bacterial taxa within the ticks changing the bacterial community in order to hinder tick vector competence.

The effective use of anti-tick microbiota vaccines in the field depends on the existence of a core tick microbiome. If the taxonomic composition of the tick microbiome is highly variable and no bacterial core is shared among individual ticks in the population, the possible harm caused by host antibodies directed against one of its bacterial components would affect only a reduced number of ticks. Several factors such as the tick species, changes in environmental traits, blood meal, host species used for blood feeding, tick immunity and developmental stage influence tick microbiome composition (Narasimhan and Fikrig 2015; Wu-Chuang et al. 2021). Accordingly, highly diverse microbiomes have been consistently detected

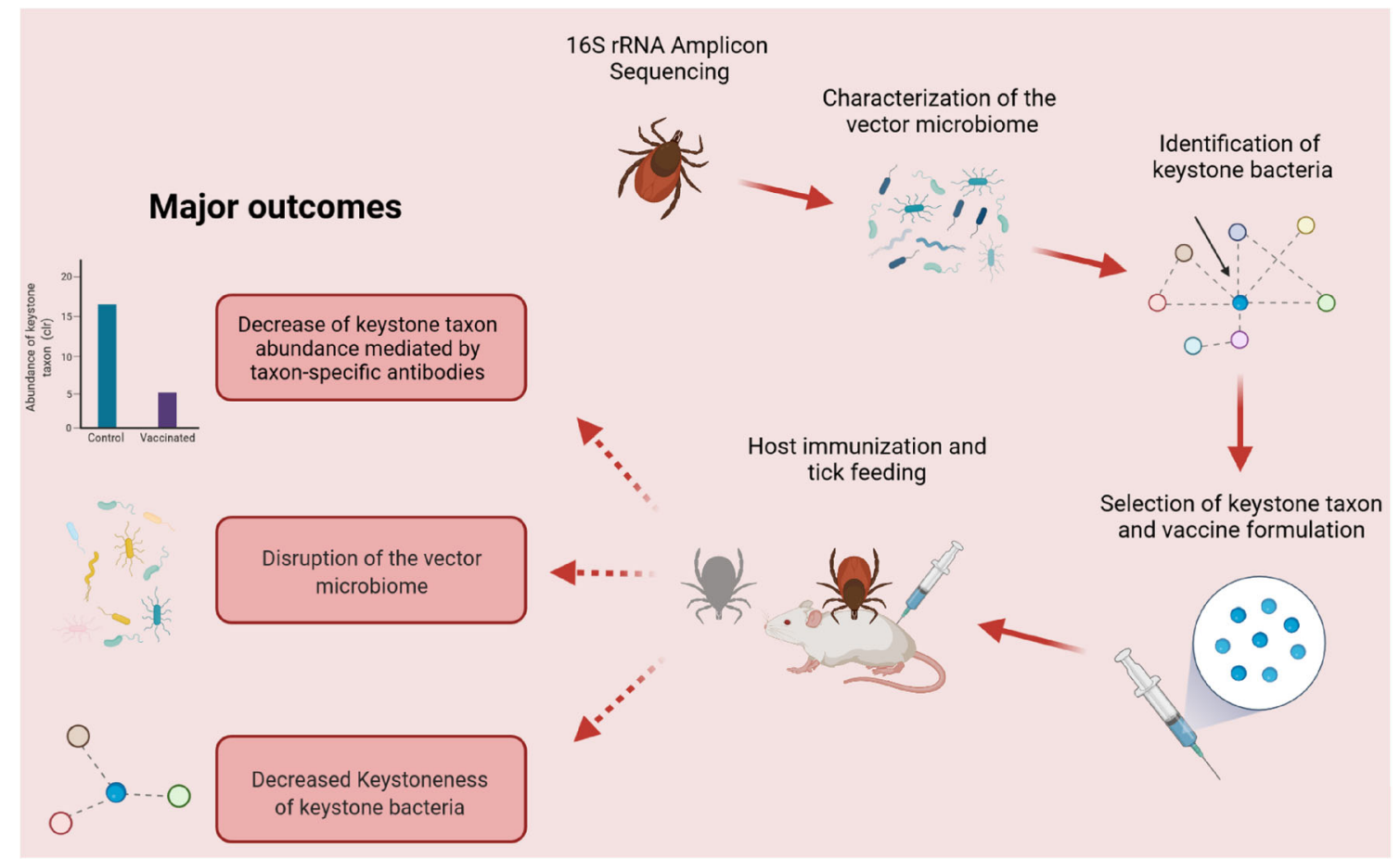

Fig. 1 Manipulation of vector microbiome with antimicrobiota vaccines. Using $16 \mathrm{~S}$ rRNA amplicon sequencing the taxonomic profiles of the tick microbiome can be characterized. Co-occurrence networks can be used to identify keystone bacteria in the microbial community. Selected keystone bacteria can be used in antimicrobiota vaccines, formulated as previously described to induce bacteria-specific antibodies in mice (MateosHernández et al. 2020, 2021). Host antibodies targeting the keystone bacteria produced three major outcomes in the tick microbial community. Created with BioRender.com 
in the midguts of different tick species (Narasimhan and Fikrig 2015; Wu-Chuang et al. 2021). In some settings the tick microbiome of individual ticks was found to be so disparate that no taxon was identified to be shared by all tested samples (e.g., Sperling et al. 2020). Other studies showed that some tick species (e.g., Ornithodoros maritimus Vermeil \& Marguet, 1967 and I. scapularis) has stable associations with bacteria of their microbiome, suggesting the existence of a core microbiome (Estrada-Peña et al. 2020a, b; Gomard et al. 2020). A taxonomic core of 61 bacterial genera (total: $821,7.4 \%$ ) was identified in the microbiome of at least one individual in groups of $I$. scapularis after disturbance by $A$. phagocytophilum infection, anti-tick vaccines, and antimicrobial peptides (Estrada-Peña et al. 2020b). Notably, 10 taxa (total: $821,1.2 \%$ ) were present in $100 \%$ of the I. scapularis samples $(n=98)$ after disturbance (Estrada-Peña et al. 2020b). This suggests that bacterial communities could develop stable associations with some tick species, which could be considered an indication of phylosymbiosis. A possible phylogenetic association between ticks and their microbiome was tested for seven different Ixodes species, including I. scapularis, Ixodes affinis (Neumann, 1899), Ixodes ovatus (Neumann, 1899), Ixodes ricinus (Linnaeus, 1758 ), Ixodes persulcatus (Schulze, 1930), Ixodes pavloskyi (Pomerantsev, 1946) and Ixodes ventalloi (Gil Collado, 1936). The congruence between dendrograms representing tick phylogeny and microbial community similarities strongly suggests a phylogenetic structure of the microbial communities associated with Ixodid ticks (Díaz-Sánchez et al. 2019). Taken together, these results validate the hypothesis that the microbiome of, at least, some tick species have stable and ubiquitous bacteria that can be used as live vaccine candidates.

Based on high eigenvector-centrality values, ubiquitousness and high relative abundance in the microbiome, two bacterial families (i.e., Enterobacteriaceae and Corynebacteriaceae) were selected as keystone taxa in the microbiome of I. scapularis and I. ricinus (MateosHernández et al. 2020). Immunization with the Enterobacteriaceae bacterium Escherichia coli elicited an anti-E. coli IgM and IgG antibody response associated with increased engorgement of I. ricinus nymphs that fed on C57BL/ 6 mice and high mortality in ticks that fed on $\alpha-1,3-$ galactosyltransferase $(\alpha 1,3 G T)$-deficient C57BL/6 mice compared with the ticks that fed on the control group, immunized with a mock formulation of PBS and adjuvant (MateosHernández et al. 2020). The antibody response of C57BL/6 and $\alpha 1,3 G T$-deficient mice differs in the ability of the later mouse strain to produce high antibody titers against the carbohydrate $\alpha$-Gal (Posekany et al. 2002; Yilmaz et al. 2014; Mateos-Hernández et al. 2020). The high mortality observed in ticks that fed on $\alpha 1,3 G T$-deficient mice could be associated with the recognition of the glycan $\alpha$-Gal on the surface of tick microbiota bacteria by lytic anti- $\alpha$-Gal host antibodies. The presence and distribution of $\alpha 1,3 G T$ genes in the tick microbiome were predicted (Mateos-Hernández et al. 2020) using the functional metagenomic inference tool PICRUSt2 (Douglas et al. 2020), and validated by PCR (MateosHernández et al. 2020). Several $\alpha 1,3 G T$ genes including gspA, waaL, waaO, waaJ, and waaR, waaT, were traced to 22 and 11 bacterial families in the I. scapularis and I. ricinus microbiome, respectively (Mateos-Hernández et al. 2020). The results suggest that host antibodies targeting gene and/or enzyme products broadly distributed in the tick gut microbiome can cause high mortality in the ticks.

\section{Dominant endosymbionts: the achilles heel of the ticks?}

In addition to a variable microbiome, next generation sequencing studies have revealed that adult female ticks are frequently dominated by a single taxon with a high relative abundance, likely endosymbionts (Hawlena et al. 2013; Ponnusamy et al. 2014; van Treuren et al. 2015; Ross et al. 2018; Chicana et al. 2019; Couper et al. 2019; Brinkerhoff et al. 2020; Guizzo et al. 2020). In contrast to the composition of the microbiome that is strongly linked to the ecological niche occupied by the ticks (Wu-Chuang et al. 2021), a high rate of transovarial transmission has been reported for tick endosymbionts in several tick species (Macaluso et al. 2001; Moore et al. 2018; Hauck et al. 2020). Some of the dominant taxa include Rickettsia detected in I. affinis (van Treuren et al. 2015), A. americanum (Ponnusamy et al. 2014), and I. scapularis female ticks (Hawlena et al. 2013; Brinkerhoff et al. 2020) or an unknown genus of the family Enterobacteriaceae identified in I. scapularis. Zhang et al. (2020) found that the dominant bacterial genus in Dermacentor silvarum (Olenev, 1931) females was Coxiella and Díaz-Sánchez et al. (2021) reported that Hyalomma lusitanicum (Koch, 1844) was dominated by Francisella-like endosymbiont (FLE). Notably, the mentioned Coxiella, Rickettsia and Francisella species were suggested to be nutritional endosymbionts in A. americanum and Rhipicephalus turanicus Pomerantsev, 1936 (Smith et al. 2015), Ixodes pacificus (Cooley \& Kohls, 1943; Hunter et al. 2015) and Amblyomma maculatum (Koch, 1844) and Ornithodoros moubata (Murray, 1877; Gerhart et al. 2016, 2018), respectively. Some of these bacteria might provide cofactors and vitamins B (e.g., Coxiella and Francisella), amino acids and heme (e.g., Francisella), or de novo-synthesized folate (e.g., Rickettsia) to the ticks. Recently Francisella endosymbionts were shown to complement the nutritional deficiency of vitamin B in the blood meal of O. moubata (Duron et al. 2018). Antibiotic-based elimination of Francisella endosymbiont from tick offspring produced anomalies in tick development and hampered nymph growth and molting to adults (Duron 
et al. 2018). Thus, transgenerational microbial inheritance in ticks includes bacteria that are indispensable for tick development. This opens the possibility of including dominant endosymbionts in antimicrobiota vaccine formulations for the control of ticks. In addition, proteins of major metabolic pathways in tick endosymbionts can be identified and used as recombinant vaccines to eliminate endosymbionts. We hypothesize that vaccination against endosymbionts may have an effect similar to antibiotics treatment in ticks. For example, the weight of $I$. ricinus nymphs fed on E. coli-immunized C57BL/6 mice increased compared with the control group (Mateos-Hernández et al. 2020, 2021), an effect also observed in I. scapularis larvae that engorged on gentamicin-treated $\mathrm{C} 3 \mathrm{H} / \mathrm{HeJ}$ mice (Narasimhan et al. 2014). Gentamicin is active against Gram-negative bacteria of the family Enterobacteriaceae. However, in addition to species of the family Enterobacteriaceae, this antibiotic is active against a wide range of other bacterial species. Therefore, it cannot be concluded that $E$. coli vaccination and gentamicin treatment increased tick weight by the same mechanism.

Most tick endosymbionts have been located in the tick ovaries and from this organ they can access the eggs. Then, the question arises as to whether the host antibodies can access the location of endosymbionts within ticks, lyse them and thus affect the physiological parameters of the tick. During feeding, ticks ingest large amount of blood containing immune proteins of the host such as antibodies and components of the complement system. In the gut lumen, host antibodies (Willadsen 1997) and complement (Rathinavelu et al. 2003) are still active and can traverse the tick gut epithelium (Ackerman et al. 1981; Ben-Yakir et al. 1987; Wang and Nuttall 1994), reaching the hemolymph (Ben-Yakir et al. 1987), accessing tick ovaries and eggs (Galay et al. 2018) as well as salivary glands and be secreted back to the host (Wang and Nuttall 1994). Intact host C3 was present in the blood meal and full-length and cleaved $\mathrm{C} 3$ were observed within the nymph (Rathinavelu et al. 2003). Furthermore, the antibodies induced against particular tick proteins react with the corresponding tick tissue and protein. For example, host antibodies against $\mathrm{Bm} 86$, a glycoprotein predominantly located in the membrane of tick gut cells (Gough and Kemp 1993), bind to the surface of epithelial cells in the tick intestine (Willadsen 1997) causing cell lysis and reducing reproductive efficiency of engorged females (Willadsen 1997). Therefore, it can be presumed that when ingested during blood feeding, the antitick microbiota antibodies could interfere with the

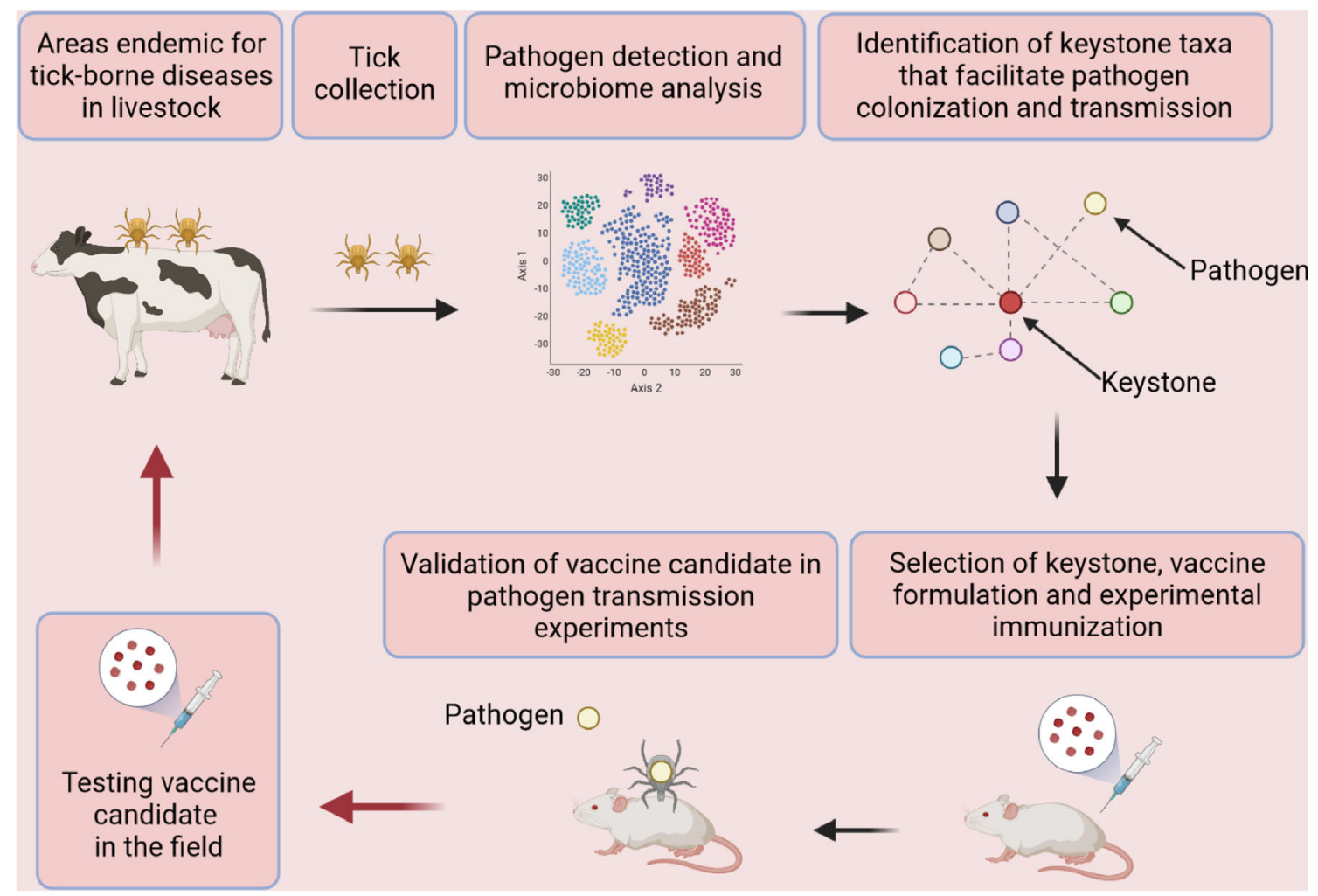

Fig. 2 Proposed workflow to develop antimicrobiota vaccines for the control of tick-borne pathogens in livestock. Ticks collected in areas endemic for tick-borne pathogens can be processed for $16 \mathrm{~S}$ rRNA amplicon sequencing to characterize the taxonomic profiles of the microbiome in infected ticks. Co-occurrence networks can be used to identify keystone bacteria potentially involved in facilitation of pathogen infection. Selected keystone bacteria can be used in antimicrobiota vaccines, to be formulated and tested in the lab for antigenicity and blocking of pathogen transmission. Selected vaccine candidates can be used in the field for the control of tick-borne pathogens. Created with BioRender.com 
physiological functionality of microbes within the ticks. A special case to consider is that Candidatus Midichloria mitochondrii, an intracellular bacterium with the unique ability to reside in the mitochondria of infected tick cells (Sassera et al. 2006). Access of host antibodies to tick cells mitochondria may be limited, thought this has not been tested.

\section{Concluding perspective}

Tick microbiome has been shown to impact tick physiology and pathogen colonization of tick tissues and transmission to the hosts. Accordingly, hereby we suggest that tick microbiome manipulation can be used for the control of tick populations and tick-borne pathogens. Recently, anti-tick microbiota vaccine has been presented as a microbiological precision tool for the modulation of tick microbiome. Targeting specific tick microbial taxa by host antibodies, acquired during feeding, successfully demonstrated that anti-tick microbiota vaccine can impact tick performance (Mateos-Hernández et al. 2020) and modulate the tick microbiome in a taxonspecific manner (Mateos-Hernández et al. 2021). Therefore, anti-tick microbiota vaccines are a new and suitable strategy for experimental manipulation of tick microbiota and subsequent alteration of tick physiology. Since endosymbionts have been widely associated with important roles in tick homeostasis, we propose that using endosymbionts in live vaccine formulations may have a great impact in tick survival and may allow the control of tick population. Furthermore, anti-tick microbiota vaccine can be employed to target keystone taxa of vector microbiota and thus, alter the bacterial community to create a dysbiosed environment that may impair pathogen colonization and its transmission to hosts (Fig. 2). Moreover, as anti-tick microbiota vaccines offer the possibility to target specific bacteria, they can be used as a tool for the study of the contribution of individual bacterial species to tick physiology. Future experiments should be directed toward understanding the mechanisms underlying anti-tick microbiota vaccines activity and their scope as a precision tool in the context of the study of tick microbiome, the control of tick population and vector competence.

Funding UMR BIPAR is supported by the French Government's Investissement d'Avenir program, Laboratoire d'Excellence "Integrative Biology of Emerging Infectious Diseases" (grant no. ANR10-LABX-62-IBEID). Alejandra Wu-Chuang is supported by Programa Nacional de Becas de Postgrado en el Exterior "Don Carlos Antonio López" (grant no. 205/2018)

\section{Declarations}

Conflict of interest The authors declare no conflict of interest.
Ethical approval Not applicable.

Informed consent Not applicable.

\section{References}

Abraham NM, Liu L, Jutras BL et al (2017) Pathogen-mediated manipulation of arthropod microbiota to promote infection. Proc Natl Acad Sci USA 114:E781-E790. https://doi.org/10.1073/pnas. 1613422114

Ackerman S, Clare FB, McGill TW, Sonenshine DE (1981) Passage of host serum components, including antibody, across the digestive tract of Dermacentor variabilis (Say). J Parasitol 67:737-740. https://doi.org/10.2307/3280459

Agler MT, Ruhe J, Kroll S et al (2016) Microbial hub taxa link host and abiotic factors to plant microbiome variation. PLoS Biol 14:1-31. https://doi.org/10.1371/journal.pbio.1002352

Banerjee S, Kirkby CA, Schmutter D et al (2016) Network analysis reveals functional redundancy and keystone taxa amongst bacterial and fungal communities during organic matter decomposition in an arable soil. Soil Biol Biochem 97:188-198. https://doi.org/10. 1016/j.soilbio.2016.03.017

Banerjee S, Walder F, Büchi L et al (2019) Agricultural intensification reduces microbial network complexity and the abundance of keystone taxa in roots. ISME J 13:1722-1736. https://doi.org/10.1038/ s41396-019-0383-2

Banerjee S, Schlaeppi K, Van der Heijden MGA (2019) Reply to "Can we predict microbial keystones? Nat Rev Microbiol 17:194. https:// doi.org/10.1038/s41579-018-0133-X

Beiko RG, Hsiao W, Parkinson J (2018) Microbiome analysis. Methods and Protocols. Springer Science and Business Media, New York

Ben-Yakir D, Fox CJ, Homer JT, Barker RW (1987) Quantification of host immunoglobulin in the hemolymph of ticks. J Parasitol 73:669 671. https://doi.org/10.2307/3282157

Ben-Yosef M, Rot A, Mahagna M, Kapri E et al (2020) Coxiella-like endosymbiont of Rhipicephalus sanguineus is required for physiological processes during ontogeny. Front Microbiol 11:1-16. https:// doi.org/10.3389/fmicb.2020.00493

Berry D, Widder S (2014) Deciphering microbial interactions and detecting keystone species with co-occurrence networks. Front Microbiol 5:1-14. https://doi.org/10.3389/fmicb.2014.00219

Brinkerhoff RJ, Brinkerhoff RJ, Clark C et al (2020) Factors affecting the microbiome of Ixodes scapularis and Amblyomma americanum. PLoS One 15:1-20. https://doi.org/10.1371/journal.pone.0232398

Chicana B, Couper LI, Kwan JY, Tahiraj E, Swei A (2019) Comparative microbiome profiles of sympatric tick species from the far-western United States. Insects 10:1-12. https://doi.org/10.3390/ insects10100353

Clayton KA, Gall CA, Mason KL, Scoles GA, Brayton KA (2015) The characterization and manipulation of the bacterial microbiome of the Rocky Mountain wood tick, Dermacentor andersoni. Parasit Vectors 8:1-5. https://doi.org/10.1186/s13071-015-1245-z

Couper LI, Kwan JY, Ma J, Swei A (2019) Drivers and patterns of microbial community assembly in a Lyme disease vector. Ecol Evol 13:7768-7779. https://doi.org/10.1002/ece3.5361

Díaz-Sánchez S, Estrada-Peña A, Cabezas-Cruz A, de la Fuente J (2019) Evolutionary insights into the tick hologenome. Trends Parasitol 35: 725-737. https://doi.org/10.1016/j.pt.2019.06.014

Díaz-Sánchez S, Fernández AM, Habela MA et al (2021) Microbial community of Hyalomma lusitanicum is dominated by Francisella-like endosymbiont. Ticks Tick Borne Dis 12:101624. https://doi.org/10. 1016/j.ttbdis.2020.101624 
Douglas GM, Maffei VJ, Zaneveld JR et al (2020) PICRUSt2 for prediction of metagenome functions. Nat Biotechnol 38:669-688. https:// doi.org/10.1038/s41587-020-0548-6

Duron O, Morel O, Noël V et al (2018) Tick-bacteria mutualism depends on B vitamin synthesis pathways. Curr Biol 28:1896-1902. https:// doi.org/10.1016/j.cub.2018.04.038

Estrada-Peña A, Cabezas-Cruz A, Obregon D (2020a) Behind taxonomic variability: the functional redundancy in the tick microbiome. Microorganisms 8:1829. https://doi.org/10.3390/ microorganisms 8111829

Estrada-Peña A, Cabezas-Cruz A, Obregon D (2020b) Resistance of tick gut microbiome to anti-tick vaccines, pathogen infection and antimicrobial peptides. Pathogens 9:309. https://doi.org/10.3390/ pathogens 9040309

Faust K, Raes J (2012) Microbial interactions: From networks to models. Nat Rev Microbiol 10:538-550. https://doi.org/10.1038/ nrmicro2832

Fisher CK, Mehta P (2014) Identifying keystone species in the human gut microbiome from metagenomic timeseries using sparse linear regression. PLoS One 9:1-10. https://doi.org/10.1371/journal.pone. 0102451

Galay RL, Matsuo T, Hernandez EP et al (2018) Immunofluorescent detection in the ovary of host antibodies against a secretory ferritin injected into female Haemaphysalis longicornis ticks. Parasitol In 67:119-122. https://doi.org/10.1016/j.parint.2017.10.006

Gerhart JG, Moses AS, Raghavan R (2016) A Francisella-like endosymbiont in the Gulf Coast tick evolved from a mammalian pathogen. Sci Rep 6:1-6. https://doi.org/10.1038/srep33670

Gerhart JG, Dutcher HA, Brenner AE et al (2018) Multiple acquisitions of pathogen-derived Francisella endosymbionts in soft ticks. Genome Biol Evol 10:607-615. https://doi.org/10.1093/gbe/evy021

Gomard Y, Flores O, Vittecoq M et al (2020) Changes in bacterial diversity, composition and interactions during the development of the seabird tick Ornithodoros maritimus (Argasidae). Micro Ecol 81: 770-783. https://doi.org/10.1007/s00248-020-01611-9

Gough JM, Kemp DH (1993) Localization of a low abundance membrane protein (Bm86) on the gut cells of the cattle tick Boophilus microplus by immunogold labeling. J Parasitol 79:900-907

Guizzo MG, Neupane S, Kucera M et al (2020) Poor unstable midgut microbiome of hard ticks contrasts with abundant and stable monospecific microbiome in ovaries. Front Cell Infect Microbiol 10:211. https://doi.org/10.3389/fcimb.2020.00211

Hauck D, Jordan D, Springer A et al (2020) Transovarial transmission of Borrelia spp., Rickettsia spp. And Anaplasma phagocytophilum in Ixodes ricinus under field conditions extrapolated from DNA detection in questing larvae. Parasit Vectors 13:176. https://doi.org/10. 1186/s13071-020-04049-7

Hawlena H, Rynkiewicz E, Toh E et al (2013) The arthropod, but not the vertebrate host or its environment, dictates bacterial community composition of fleas and ticks. ISME J 7:221-223. https://doi.org/ 10.1038 /ismej.2012.71

Ho A, Mendes LW, Lee HJ et al (2020) Response of a methane-driven interaction network to stressor intensification. FEMS Microbiol Ecol 96:1-13. https://doi.org/10.1093/femsec/fiaa180

Hunter DJ, Torkelson JL, Bodnar J et al (2015) The rickettsia endosymbiont of Ixodes pacificus contains all the genes of de novo folate biosynthesis. PLoS One 10:1-15. https://doi.org/10.1371/journal. pone. 0144552

Kwan JY, Griggs R, Chicana B, Miller C, Swei A (2017) Vertical vs. horizontal transmission of the microbiome in a key disease vector, Ixodes pacificus. Mol Ecol 26:6578-6589. https://doi.org/10.1111/ mec. 14391

Landesman WJ, Mulder K, Fredericks LP, Allan BF (2019) Crosskingdom analysis of nymphal-stage Ixodes scapularis microbial communities in relation to Borrelia burgdorferi infection and load.
FEMS Microbiol Ecol 95:1-14. https://doi.org/10.1093/femsec/ fiz167

Layeghifard M, Hwang DM, Guttman DS (2017) Disentangling interactions in the microbiome: a network perspective. Trends Microbiol 25:217-228. https://doi.org/10.1016/j.tim.2016.11.008

Macaluso KR, Sonenshine DE, Ceraul SM, Azad AF (2001) Infection and transovarial transmission of rickettsiae in Dermacentor variabilis ticks acquired by artificial feeding. Vector Borne Zoonot Dis 1:45-53. https://doi.org/10.1089/153036601750137660

Mateos-Hernández L, Obregon D, Maye J et al (2020) Anti-tick microbiota vaccine impacts Ixodes ricinus performance during feeding. Vaccines 8:1-21. https://doi.org/10.3390/vaccines8040702

Mateos-Hernández L, Obregon D, Wu-Chuang A et al (2021) Antimicrobiota vaccines modulate the tick microbiome in a taxonspecific manner. BioRxiv. https:/www.biorxiv.org/content/, https://doi.org/10.1101/2021.05.12.443756v1

Moore TC, Pulscher LA, Caddell L et al (2018) Evidence for transovarial transmission of tick-borne rickettsiae circulating in Northern Mongolia. PLoS Negl Trop Dis 12:e006696. https://doi.org/10. 1371/journal.pntd.0006696

Narasimhan S, Fikrig E (2015) Tick microbiome: The force within. Trends Parasitol 31:315-323. https://doi.org/10.1016/j.pt.2015.03. 010

Narasimhan S, Rajeevan N, Liu L et al (2014) Gut microbiota of the tick vector Ixodes scapularis modulate colonization of the Lyme disease spirochete. Cell Host Microbe 15:58-71. https://doi.org/10.1016/j. chom.2013.12.001

Narasimhan S, Swei A, Abouneameh S, Pal U, Pedra JHF, Fikrig E (2021) Grappling with the tick microbiome. Trends Parasitol S1471-4922(21):00084-2. https://doi.org/10.1016/j.pt.2021.04.004

Newman MEJ (2010) An introduction to the mathematical tools used in the study of networks, tools that will be important to many subsequent developments In: Newman M (ed) Networks: An Introduction. Oxford, pp 109-167. https://doi.org/10.1093/acprof: oso/9780199206650.003.0006

Ponnusamy L, Gonzalez A, Van Treuren W et al (2014) Diversity of rickettsiales in the microbiome of the lone star tick, Amblyomma americanum. Appl Environ Microbiol 80:354-359. https://doi.org/ 10.1128/AEM.02987-13

Posekany KJ, Pittman HK, Bradfield JF, Haisch CE, Verbanac KM (2002) Induction of cytolytic anti-Gal antibodies in alpha-1,3galactosyltransferase gene knockout mice by oral inoculation with Escherichia coli O86:B7 bacteria. Infect Immun 70:6215-6222. https://doi.org/10.1128/IAI.70.11.6215-6222.2002

Rathinavelu S, Broadwater A, De Silva AM (2003) Does host complement kill Borrelia burgdorferi within ticks? Infect Immun 71:822829. https://doi.org/10.1128/IAI.71.2.822-829.2003

Ross BD, Hayes B, Radey MC et al (2018) Ixodes scapularis does not harbor a stable midgut microbiome. ISME J 12:2596-2607. https:// doi.org/10.1038/s41396-018-0161-6

Röttjers L, Faust K (2019) Can we predict keystones? Natu Rev Microbiol 17:193. https://doi.org/10.1038/s41579-018-0132-y

Ruhnau B (2000) Eigenvector-centrality - a node-centrality ? Soc Networks 22:357-365. https://doi.org/10.1016/S0378-8733(00) 00031-9

Sassera D, Beninati T, Bandi C et al (2006) 'Candidatus Midichloria mitochondrii', an endosymbiont of the tick Ixodes ricinus with a unique intramitochondrial lifestyle. Int J Syst Evol Microbiol 56(Pt 11):2535-2540. https://doi.org/10.1099/ijs.0.64386-0

Smith TA, Driscoll T, Gillespie JJ, Raghavan R (2015) A Coxiella-like endosymbiont is a potential vitamin source for the lone star tick. Genome Biol Evol 7:831-838. https://doi.org/10.1093/gbe/evv016

Sperling J, MacDonald Z, Normandeau J, Merrill E, Sperling F, Magor K (2020) Within-population diversity of bacterial microbiomes in winter ticks (Dermacentor albipictus). Ticks Tick Borne Dis 11(6): 101535. https://doi.org/10.1016/j.ttbdis.2020.101535 
van der Heijden MGA, Hartmann M (2016) Networking in the plant microbiome. PLoS Biol 14:1-9. https://doi.org/10.1371/journal. pbio. 1002378

Van Treuren W, Ponnusamy L, Brinkerhoff RJ et al (2015) Variation in the microbiota of Ixodes ticks with regard to geography, species, and sex. Appl Environ Microbiol 81:6200-6209. https://doi.org/10. 1128/AEM.01562-15

Wang H, Nuttall PA (1994) Excretion of host immunoglobulin in tick saliva and detection of Igg-binding proteins in tick haemolymph and salivary glands. Parasitology 109:525-530. https://doi.org/10.1017/ S0031182000080781

Willadsen P (1997) Novel vaccines for ectoparasites. Vet Parasitol 71: 209-222. https://doi.org/10.1016/S0304-4017(97)00028-9

Wu-Chuang A, Hodžić A, Mateos-Hernández L et al (2021) Current debates and advances in tick microbiome research. CRPVBD. https://doi.org/10.1016/j.crpvbd.2021.100036

Yang X, Koči J, Smith AA et al (2021) A novel tick protein supports integrity of gut peritrophic matrix impacting existence of gut microbiome and Lyme disease pathogens. Cell Microbiol 23: e13275. https://doi.org/10.1111/cmi.13275

Yilmaz B, Portugal S, Tran TM et al (2014) Gut microbiota elicits a protective immune response against malaria transmission. Cell 159:1277-1289. https://doi.org/10.1016/j.cell.2014.10.053
Ze X, Duncan SH, Louis P, Flint HJ (2012) Ruminococcus bromii is a keystone species for the degradation of resistant starch in the human colon. ISME J 6:1535-1543. https://doi.org/10.1038/ismej.2012.4

Zhang CM, Li NX, Zhang TT et al (2017) Endosymbiont CLS-HI plays a role in reproduction and development of Haemaphysalis longicornis. Exp Appl Acarol 73:429-438. https://doi.org/10.1007/ s10493-017-0194-y

Zhang R, Yu G, Huang Z, Zhang Z (2020) Microbiota assessment across different developmental stages of Dermacentor silvarum (Acari: Ixodidae) revealed stage-specific signatures. Ticks Tick Borne Dis 11:101321. https://doi.org/10.1016/j.ttbdis.2019.101321

Zheng H, Yang T, Bao Y et al (2021) Network analysis and subsequent culturing reveal keystone taxa involved in microbial litter decomposition dynamics. Soil Biol Biochem 157:108230. https://doi.org/10. 1016/j.soilbio.2021.108230

Zhong J, Jasinskas A, Barbour AG (2007) Antibiotic treatment of the tick vector Amblyomma americanum reduced reproductive fitness. PLoS One 2:1-7. https://doi.org/10.1371/journal.pone.0000405

Publisher's note Springer Nature remains neutral with regard to jurisdictional claims in published maps and institutional affiliations. 